Revista lus et Praxis, Año 17, No 1, 2011, pp. 231 - 246

ISSN 0717 - 2877

Universidad de Talca - Facultad de Ciencias Jurídicas y Sociales

"Para escribir una tesis jurídica: técnicas de investigación en Derecho"

Sebastián López Escarcena

\title{
PARA ESCRIBIR UNA TESIS JURÍDICA: TÉCNICAS DE INVESTIGACIÓN EN DERECHO*
}

\author{
TO WRITE A LEGAL THESIS: ReSEARCH TECHNIQUES IN LAW
}

Sebastián López Escarcena ${ }^{* *}$

\begin{abstract}
RESUMEN
Investigar es buscar información para responder una pregunta destinada a resolver un problema. Esta actividad requiere un método, que puede definirse como un modo de decir o de hacer algo con orden. En derecho, normalmente, será necesario plantear una proposición razonada o tesis, orientada a hacer una contribución original.

Es indispensable definir el objeto de la tesis a fin de determinar la accesibilidad y manejo de las fuentes, tanto primarias como secundarias. Elegido el tema de la tesis, se debe hacer una bibliografía e índice tentativos. El plan de trabajo incluye un título provisorio, que transformado en pregunta guiará la investigación. Su respuesta se debe articular en torno a un argumento central, siguiendo cuatro modelos posibles: descriptivo; analítico; argumentativo, y mixto. Redactar una respuesta efectiva a una pregunta de investigación requiere respetar ciertas reglas que rigen su macro y/o micro-estructuras. Un aspecto fundamental en este proceso es la satisfacción de las expectativas del lector.
\end{abstract}

ABSTRACT

Research means to look for information to answering a question aimed at solving a problem. This activity requires a method, which can be defined as a form of saying or doing something with order. In law, it will normally be necessary to state a reasoned proposition or thesis, destined to make an original contribution. It is essential to define the object of the thesis in order to establish the accessibility and handling of the sources, both primary and secondary. Once the subject of the thesis is chosen, a tentative bibliography and table of contents should be made. The work plan includes

\footnotetext{
*El autor agradece las observaciones de Jaime Alcalde Silva, Nicolás Cobo Romaní, Paulina González Vergara y Sebastián Zárate Rojas, todos profesores de la Facultad de Derecho de la Pontificia Universidad Católica de Chile. También agradece los comentarios de sus alumnos del curso sobre Introducción a la investigación jurídica que imparte en el programa de Doctorado en Derecho de la misma Universidad. Cualquier error en el artículo, es responsabilidad exclusiva del autor. Trabajo recibido el 8 de octubre y aprobado el 11 de noviembre de 2010.

** Profesor de Derecho Internacional e investigador de la Facultad de Derecho de la Pontificia Universidad Católica de Chile; Profesor visitante (visiting fellow), Universidad Católica de Lovaina, Bélgica; Doctor en Derecho (PhD), Universidad de Edimburgo, Reino Unido; Magíster en Derecho (LLM), Universidad de Leiden, Países Bajos; Abogado; Licenciado en Derecho, Pontificia Universidad Católica de Chile. Correo electrónico: rlopeze@uc.cl.
} 
a provisional title, which transformed in a question will guide the research. Its answer should be articulated around a central argument, following four possible models:

descriptive; analytic; argumentative; and matrix patterns. Drafting an effective answer to a research question requires respecting certain rules that regulate its macro and/ or micro-structures. A fundamental aspect of this process is the satisfaction of the reader's expectations.

PALABRAS CLAVE Investigación Jurídica, Metodología, Tesis

KEY WORDS Legal Research, Methodology, Thesis

\section{INTRODUCCIÓN}

En sus Instrucciones para olvidar el Quijote, Fernando Savater nos da una recomendación peculiar: para leer este libro -dice- lo primero que hay que hacer es olvidar a su personaje principal. ${ }^{1}$ ¿Qué quiere decir con esto? Simplemente, que la única forma de acercarse a la novela de Cervantes es dejando de lado las ideas preconcebidas que tenemos de Alonso Quijano, provenientes de las diversas interpretaciones, simplificaciones y deformaciones que se han hecho de él por siglos. La misma recomendación se le puede dar a alguien que se enfrenta por primera vez a una investigación jurídica. Hay que olvidar los manuales y tratados de derecho tradicionales de nuestro medio. Entiéndase bien: no se trata de desatender lo que dicen esos manuales y tratados, sino de evitar partir de la extendida y errada idea de que los textos jurídicos tienen que ser necesariamente mamotretos ilegibles y aburridos. Así como a los libros no se les debe juzgar por su tapa, tampoco corresponde valorarlos por su volumen o por su peso. Lo que importa es su contenido, cuya calidad dependerá de lo siguiente: que el texto resultante dé una respuesta efectiva a la pregunta principal planteada. En otras palabras, el autor tendrá que satisfacer las expectativas del lector, que él mismo ha creado.

Las reflexiones que vienen a continuación no pretenden ser un estudio acabado de metodología de la investigación jurídica. ${ }^{2}$ Su objetivo es más modesto:

\footnotetext{
${ }^{1}$ Ver SAVATER, Fernando, Instrucciones para olvidar el Quijote, Editorial Santillana, Madrid, 1995, pp. 17-31.

${ }^{2}$ La bibliografía al respecto es amplia y su calidad es disímil. Entre la reciente, en español, ver e.g. LARA Sáenz, Leoncio, Procesos de investigación jurídica, UNAM, México D.F., 1991; WiTkER, Jorge, Cómo elaborar una tesis en derecho, Editorial Civitas, Madrid, 1991; Martínez Pichardo, José, Lineamientos para la investigación jurídica, Editorial Porrúa, México D.F., 1998; Arellano García, Carlos, Métodos y técnicas de la investigación jurídica, Editorial Porrúa, México D.F., 1999; HeRRERA, Enrique, Práctica metodológica de la investigación jurídica, Editorial Astrea, Buenos Aires, 2002; Hernández Estévez, Sandra; López Durán, Rosalío, Técnicas de investigación jurídica, Oxford University Press, México D.F., 2002; Scavone, Graciela, Cómo se escribe una tesis, Editorial La Ley, Buenos Aires, 2002; Álvarez Undurraga, Gabriel, Curso de investigación jurídica, Editorial LexisNexis, Santiago de Chile, 2005; Ramos Núñez, Carlos, Cómo hacer
} 
servir como herramienta a quien quiera o necesite dar una respuesta estructurada, lo más completa posible, a una pregunta de derecho. En otras palabras, ayudar a quien vaya a emprender una investigación y que no sepa cómo hacerlo. Algo que nos ha pasado a todos, alguna vez.

\section{LA PREGUNTA PRINCIPAL Y EL ÍNDICE TENTATIVO}

Investigar es buscar información para responder una pregunta destinada a resolver un problema. ${ }^{3}$ Se llama investigación aplicada la que soluciona problemas prácticos, y recibe el nombre de investigación pura la que se dedica a los problemas conceptuales -i.e. a entender mejor un asunto. ${ }^{4} \mathrm{O}$, como dice el Diccionario de la Lengua de la Real Academia Española, a aumentar los conocimientos sobre una materia específica. ${ }^{5}$ Ambas clases de investigación se pueden dar en derecho. Cualquiera sea su fin, la investigación requiere un método, que no es otra cosa que un modo de decir o de hacer algo con orden. En otras palabras, para investigar se necesita seguir ciertos procedimientos. El método es propio de las ciencias y puede ser inductivo (de lo particular a lo general) o deductivo (de lo general a lo particular). Ambas clases de métodos se pueden presentar en la investigación jurídica. Los métodos que son particulares a ésta son el histórico, el dogmático, el comparativo y el empírico. ${ }^{6}$ Salvo el último, que involucra la elaboración de encuestas, el análisis de estadísticas y otros, todos se reducen a leer y escribir. Así de simple. Por tanto, mejor que elucubrar abstracciones metodológicas, es preferible referirse a técnicas de investigación jurídica, cuya eficacia variará según quién las utilice.

En Derecho, la investigación no sólo constituye la base del ejercicio de la profesión legal, sino que sirve de guía a cualquier persona que quiera o necesite interpretar y aplicar una o más normas jurídicas. Su utilidad es manifiesta o patente en la creación y modificación de éstas a través del proceso legislativo, y en su interpretación y aplicación por medio del proceso judicial. El resul-

una tesis en derecho y no envejecer en el intento, Gaceta Jurídica, Lima, 2007; Corral TalCIANI, Hernán, Cómo hacer una tesis en derecho, Editorial Jurídica de Chile, Santiago, 2008; ElGueta, María Francisca; Palma, Eric, La investigación en ciencias sociales y jurídicas, Universidad de Chile, Santiago, 2008, y Cuerda Riezu, Antonio, Cum laude. Guía para realizar una tesis doctoral en derecho, Editorial Tecnos, Madrid, 2008.

${ }^{3}$ Воотн, Wayne; Colomb, Gregory; Williams, Joseph, The Craft of Research, University of Chicago Press, Chicago, 2008, pp. 10-1, 19-20 y 35-6.

${ }^{4}$ Sobre los problemas prácticos y conceptuales ver, en general, Воотн; ColomB; Williams, The Craft, cit. nota n. 3, pp. 52-8 y 62-5.

Respecto a la diferencia entre investigación pura y aplicada, ver BoотH; ColomB; Williams, The Craft, cit. nota n. 3, pp. 59-62.

${ }^{5}$ En: http:www.rae.es [visitado el 08/10/2010].

${ }^{6}$ Ver Corral, Cómo hacer, cit. nota n. 2, pp. 55-80. 
tado de la investigación jurídica podrá tomar la forma de un manual o texto de estudio, de un comentario a una sentencia, o de una recensión a un libro. También podrá resultar en una monografía, en un artículo académico, en un ensayo o en un informe en derecho u opinión legal. En los cuatro últimos casos se requiere plantear una proposición razonada o tesis, estructurada en torno a un argumento central y orientada a hacer una contribución original. ${ }^{7}$ Esto es, decir algo que todavía no se ha dicho, o revisar desde una perspectiva distinta lo que ya se ha dicho. ${ }^{8}$ Umberto Eco señala que una tesis estudia un objeto valiéndose de determinados instrumentos. ${ }^{9}$ En derecho, el objeto son libros o documentos -la fuente primaria-, y los instrumentos son otros libros o documentos -las fuentes secundarias o literatura crítica. Por decirlo de otra manera, los libros de los que se habla y los libros con la ayuda de los cuales se habla. ${ }^{10}$ Recurrir a las fuentes primarias es ineludible. Aun cuando los instrumentos las reproduzcan, éstos siguen siendo fuentes de segunda mano. Es indispensable, por tanto, definir cuanto antes el objeto de la tesis, a fin de determinar la accesibilidad de las fuentes, tanto primarias como secundarias. ${ }^{11}$ Eco recomienda elegir un tema si se sabe dónde ubicar las fuentes; es fácil llegar a ellas, y se está capacitado para manejarlas. ${ }^{12}$

El manejo de fuentes se relaciona con el conocimiento de otros idiomas. Al respecto, la exigencia variará según el tema elegido. Por ejemplo, si se inves-

\footnotetext{
7 Ver Murray, Rowena, How to Write a Thesis, Open University Press, Maidenhead, 2009, p. 107. La palabra "tesis" también se utiliza para designar al texto resultante del trabajo de investigación principal de un alumno universitario. MurRay, How to Write, recién citado, p. 123.

${ }^{8}$ Ver Eco, Umberto, Cómo se hace una tesis. Técnicas y procedimientos de investigación, estudio y escritura, Editorial Gedisa, Barcelona, 1993, pp. 20 y 49. Ver, también, Dunleavy, Patrick, Authoring a PhD. How to Plan, Draft, Write and Finish a Doctoral Thesis or Dissertation, Palgrave Macmillan, Nueva York, 2003, p. 27; Germano, William, From Dissertation to Book, University of Chicago Press, Chicago, 2005, p. 17-21; Cuerda Riezu, Cum laude, cit. nota n. 2, pp. 101 y 103-4; Murray, How to Write a Thesis, cit. nota n. 7, pp. 59-61; y RugG, Gordon; Petre, Marian, The Unwritten Rules of PhD Research, Open University Press, Maidenhead, 2009, pp. 96-97. Una tesis de compilación, donde el estudiante se limita a demostrar que ha revisado críticamente la mayor parte de la literatura existente, que ha sido capaz de exponerla con claridad y que ha intentado interrelacionar los diversos puntos de vista, ofreciendo una panorámica inteligente, no es suficiente en una investigación doctoral. Tiene sentido a nivel de licenciatura, pero sólo si no existe todavía una tesis parecida en ese tema. Al respecto ver Eco, Cómo se hace, cit. nota n. 8, pp. 20-1 y 50.

${ }^{9}$ Eco, Cómo se hace, cit. nota n. 8, p. 69.

${ }^{10}$ Eco, Cómo se hace, cit. nota n. 8, p. 135. Ver, también, Bоoтн; Colomb; Wilıams, The Craft, cit. nota n. 3, pp. 68-70.

${ }^{11}$ Ver Eco, Cómo se hace, cit. nota n. 8, pp. 70-71. E.g. de fuentes secundarias son las traducciones, las antologías y los informes elaborados por otros autores del o los libros que sirven de fuente primaria. Eco, Cómo se hace, cit. nota n. 8, p. 75.

${ }^{12}$ Eco, Cómo se hace, cit. nota n. 8, p. 71. Ver, también, Bоотн; Colomb; Wiluams, The Craft, cit. nota n. 3, p. 32.
} 
tiga en derecho romano o canónico, leer en latín será inevitable; si se trata de derecho civil, se requerirá entender francés e italiano y si el tema es de derecho penal, será indispensable tener conocimientos de alemán. En cualquiera de estos casos, será igualmente necesario poder leer en inglés. ${ }^{13}$ Este idioma será también esencial al abordar temas de derecho comercial e internacional. Cualquiera sea el caso, es preciso elegir una tesis que no implique el conocimiento de lenguas que no se manejen, y que uno no esté dispuesto a aprender. ${ }^{14}$ Una vez elegido el tema de la tesis, se debe comenzar por hacer una bibliografía y un índice tentativos. Un buen comienzo es el catálogo de materias. Reunidos algunos libros que resulten útiles, se puede recurrir a sus bibliografías para encontrar más libros o artículos sobre la materia. Y así, sucesivamente. Al mismo tiempo, se puede recurrir al catálogo de autores, que es más exacto que el de materias, puesto que no depende de las interpretaciones del bibliotecario. ${ }^{15}$ Obviamente, no es posible leer todos los libros o artículos encontrados, de buenas a primeras. Eco se pregunta en qué orden hay que leer estos libros o artículos. En su opinión, conviene abordar de inmediato dos o tres textos de literatura crítica elegidos entre los más generales, a fin de tener una idea del tema a tratar. A continuación, se debe enfrentar directamente la fuente primaria. Luego habrá que volver al resto de la literatura crítica. ${ }^{16}$ Pero éste es sólo un consejo. En la práctica, cada investigador tendrá su propia forma de acercarse a las fuentes primaria y secundaria.

Teniendo una bibliografía inicial, se debe elaborar un índice provisional: éste constituirá el plan de trabajo. Tendrá, por tanto, que ser definido lo antes posible. El índice tentativo será reestructurado varias veces a lo largo de la investigación, por lo que conviene hacer un breve resumen de cada uno de sus capítulos. ${ }^{17}$ El plan de trabajo incluye el título tentativo o provisional de

\footnotetext{
${ }^{13}$ Ver Corral, Cómo hacer, cit. nota n. 2, p. 94.

${ }^{14} \mathrm{ECO}$, Cómo se hace, cit. nota n. 8, p. 43.

${ }^{15}$ Ver Eco, Cómo se hace, cit. nota n. 8, pp. 80-81. Ver, también, Corral, Cómo hacer, cit. nota n. 2, pp. 97-98; Bоотн; COlomb; WillaAms, The Craft, cit. nota n. 3, pp. 70-81, y CUeRDA, Cum laude, cit. nota n. 2, pp. 44-45 y 141-147. La forma de citar los libros y artículos referidos dependerá de las normas aplicables que tenga al efecto la institución, revista académica o editorial respectiva. Sobre las normas de citación y referencia en derecho ver, en general, Corral, Cómo hacer, cit. nota n. 2, pp. 141-166, y Cuerda, Cum laude, cit. nota n. 2, pp. 106-108. Ver, también, el Oxford Standard for Citation of Legal Scholars. En: http://denning.law.ox.ac.uk/published/oscola.shtml [visitado el 08/10/2010].

En caso del derecho internacional, ver e.g. las instrucciones para colaboradores del Leiden Journal of International Law. En: http://assets.cambridge.org/LJL/LJL_ifc.pdf [visitado el 08/10/2010].

${ }^{16}$ Eco, Cómo se hace, cit. nota n. 8, pp. 133 y 135.

Ver también Cuerda, Cum laude, cit. nota n. 2, pp. 55-56. Respecto al manejo de las fuentes, ver en general Воотн; Colomв; Williams, The Craft, cit. nota n. 3, pp. 84-100.

17 Ver Cuerda, Cum laude, cit. nota n. 2, pp. 69-70. Ver, también, MurRay, How to Write, cit. nota n. 7, p. 125.
} 
la tesis. Dicho título, transformado en pregunta, se convertirá en parte esencial de la investigación. ${ }^{18}$ Según Patrick Dunleavy, se debe evitar elegir el siguiente tipo de preguntas: aquellas que exceden la posibilidad de respuesta de alguien sin mayor experiencia en investigación; aquellas extremadamente puntuales, las que sólo atraerán a un puñado de lectores, y aquellas que pretenden con su respuesta llenar una laguna en el área respectiva. ${ }^{19}$ Estas últimas existen por una razón -debido al poco interés o la extrema dificultad, del tema correspondiente. La pregunta principal tiene que formularse en torno a un problema, sea práctico o conceptual. Y tiene que hacerse de tal manera, que permita la exposición de los descubrimientos proyectados como un todo articulado. El porqué y para qué de la investigación, serán sólo el comienzo de su respuesta. ${ }^{20}$

\section{EN BUSCA DE UNA RESPUESTA}

Todo investigador es un escritor. Como tal, está sujeto a la misma regla de oro: la manera en que se escribe, importa tanto como lo que se escribe. ${ }^{21} \mathrm{El}$ desarrollo de las ideas se hace en el papel. Rara vez se piensa primero y se escribe después -ambos actos van generalmente de la mano. Las ideas cristalizan al ser escritas; es entonces cuando nos vemos forzados a tomar partido, y quedan en evidencia las flaquezas y contradicciones de nuestro pensamiento. ${ }^{22}$ Quizás al principio de la investigación la balanza se incline más por la lectura. Pero una vez que se tenga un manejo de los conceptos básicos del tema elegido, es recomendable seguir leyendo y escribiendo a la vez. ${ }^{23}$ Uno de los deberes primordiales del investigador-escritor es no olvidar nunca al destinatario de su texto. Si la investigación no ha sido solicitada por una persona o institución particular, conviene dirigir el texto a un lector imaginario medianamente informado. Una buena opción es redactar un texto que sea entendible para un académico de otra disciplina afín, por ejemplo, uno de filosofía o de ciencia política, si se investiga

\footnotetext{
${ }^{18}$ Ver ECO, Cómo se hace, cit. nota n. 8, pp. 137-9. Ver, también, Corral, Cómo hacer, cit. nota n. 2, pp. 98-100, y Rugg; Petre, The Unwritten Rules, cit. nota n. 8, p. 102.

${ }^{19}$ Ver Dunleavy, Authoring a PhD., cit. nota n. 8, pp. 20-1, 23 y 25.

${ }^{20}$ En cuanto a las preguntas de investigación ver, en general, Bоотн; Colomb; Williams, The Craft, cit. nota n. 3, pp. 40-48 y 50-52. Ver, también, RugG; Petre, The Unwritten Rules, cit. nota n. 8, pp. 146-148.

${ }^{21}$ Germano, From Dissertation, cit. nota n. 8, p. 8.

${ }^{22}$ Ver Dunleavy, Authoring a PhD., cit. nota n. 8, p. 26. Ver, también, Germano, From Dissertation, cit. nota n. 8, pp. 21-24; BоOth; Colomb; Willams, The Craft, cit. nota n. 3, pp. 11-13, 33 y 187; Corral, Cómo hacer, cit. nota n. 2, p. 127; Cuerda, Cum laude, cit. nota n. 2, p. 75, y Murray, How to Write, cit. nota n. 7, pp. 6-7.

${ }^{23}$ Ver, e.g., MurRaY, How to Write, cit. nota n. 7, pp. 2, 13 y 21.
} 
en derecho. ${ }^{24}$ Para este efecto, hay que definir todos los términos técnicos que constituyan un elemento clave en la línea de argumentación principal. ${ }^{25}$ Con su tesis, el investigador se integrará a un debate en una comunidad académica o profesional determinada. ${ }^{26}$ Como parte de dicha comunidad, tendrá que dar una opinión informada y personal sobre los aspectos más significativos de su disciplina, en la cual necesariamente va a haber distintas tendencias. Existirá una mayoritaria y habrá otras que serán minoritarias -sea porque vienen surgiendo o porque están en retirada. Todo investigador termina, inevitablemente, por acercarse a una de estas tendencias. Sin embargo, no debiera quedar neutralizado por éstas, si quiere mantener su individualidad en el mundo académico o profesional. ${ }^{27}$ Para este fin, tiene que ser independiente y pensar por sí mismo, y esto no es fácil. En todo proceso de aprendizaje hay un cierto sentimiento de vértigo. Nos alejamos de la seguridad de aquello conocido para adentramos en el incierto territorio de aquello por conocer. ${ }^{28}$ Hay que tener presente la dificultad de ser original y conformarse con pequeños pasos iniciales, que nos puedan llevar con el tiempo a recorrer grandes distancias. Se debe ser realista, pero no por eso dejar de ser ambicioso. ${ }^{29}$

A lo largo de la investigación, la pregunta principal será reformulada varias veces. Su respuesta se articulará en torno a un argumento central, cuya estructura Wayne Booth, Gregory Colomb y Joseph Williams describen de la siguiente manera. ${ }^{30}$ Todo argumento de investigación empieza -según estos autores- por una afirmación o hipótesis, la que va acompañada de las razones y la evidencia que le sirven de apoyo. ${ }^{31}$ Junto a estas razones y evidencia, el argumento tendrá que anticipar y reconocer las posibles objeciones a la

\footnotetext{
${ }^{24}$ Germano, William, Getting it Published, University of Chicago Press, Chicago, 2008, p. 3. No todos los autores están de acuerdo con lo anterior. Algunos señalan que en la redacción del texto se debiera considerar únicamente a la comunidad académica de la disciplina en cuestión. En este caso, el derecho.

Ver, e.g., Bоотн; Colomb; Willaams, The Craft, cit. nota n. 3, pp. 20-25, y Cuerda, Cum laude, cit. nota n. 2, pp. 81-84. Un error común en los doctorandos es dirigir su trabajo exclusivamente al supervisor o examinadores de la tesis.

Al respecto, ver MurRaY, How to Write, cit. nota n. 7, pp. 75-77.

${ }^{25}$ Eco, Cómo se hace, cit. nota n. 8, pp. 178 y 186.

${ }^{26}$ Ver Murray, How to Write, cit. nota n. 7, p. 29.

27 Ver Dunleavy, Authoring a PhD., cit. nota n. 8, pp. 27 y 32. Ver, también, Germano, From Dissertation, cit. nota n. 8, pp. 20-21, y CuerDA, Cum laude, cit. nota n. 2, pp. 118-119.

${ }^{28}$ Ver Baln, Ken, What the Best College Teachers Do, Harvard University Press, Cambridge-Massachusetts, 2004, pp. 26-29 y 83-85.

${ }^{29}$ Ver Dunleavy, Authoring a PhD., cit. nota n. 8, pp. 34 \& 38.

${ }^{30} \mathrm{Al}$ respecto ver, en general, Bоотн; Cоlomb; Williams, The Craft, cit. nota n. 3, pp. 105-19.

${ }^{31}$ Ver, también, Murray, How to Write, cit. nota n. 7, p. 157.
} 
afirmación hecha y dar respuesta a cada una de éstas. ${ }^{32}$ Del argumento así estructurado, se deberá desprender un principio general aplicable a otras situaciones similares, a través del método deductivo. La existencia de dicho principio explicará la relevancia de la afirmación inicial, relacionándola con las razones ofrecidas. ${ }^{33} \mathrm{Un}$ argumento articulado en estos términos se asemejará a una conversación virtual y constante entre el autor y su lector, que hará más efectivo y entretenido su texto resultante. Dunleavy distingue cuatro formas de responder la pregunta principal en una tesis en humanidades o ciencias sociales. ${ }^{34}$ La primera es el modelo descriptivo, que explica un asunto siguiendo el orden en que éste se presenta externamente. Muchas tesis utilizan este modelo. La estructura de una en derecho estará determinada, en este caso, por la secuencia histórica de los hechos-como ocurre con las tesis cronológicas- o por la organización de una ley o regulación -como sucede con las tesis que siguen el orden de un texto.

El modelo descriptivo se utiliza mucho en los trabajos de investigación de licenciatura y de magíster. Sin embargo, para que este modelo funcione en una tesis doctoral o en un libro, se requiere ser un escritor hábil. De lo contrario, se corre el riesgo que el argumento central de la tesis se pierda irremediablemente en una estructura impuesta desde fuera. La segunda forma de respuesta que distingue Dunleavy es el modelo analítico. ${ }^{35} \mathrm{~A}$ diferencia del anterior, aquí la estructura la da el propio autor. No debiera ser difícil separar y reorganizar una descripción compleja distinguiendo, por ejemplo, períodos históricos o los componentes o elementos de un problema intelectual: conceptos, teorías, etcétera. El modelo analítico tiene muchas ventajas, siempre que se elijan categorías organizativas que sean simples y sólidas a la vez. Distinciones sutiles o que toman mucho tiempo en ser explicadas, no son adecuadas para estructurar una tesis. Si se sobreanaliza el problema, el argumento central se perderá en una maraña de conceptos y teorías. Una tercera alternativa es el modelo argumentativo. Éste consiste en reunir todos los puntos que puedan incluirse en una interpretación o posición intelectual determinada y exponerlos coherentemente, repitiendo a continuación este proceso respecto de la interpretación o posición contraria. El modelo argumentativo se verá bien organizado, si se distinguen posiciones intelectuales claras y se utilizan denominaciones ya reconocidas en doctrina. El problema con este modelo radica en que la estructura argumento-

\footnotetext{
${ }^{32}$ Ver, e.g., Murray, How to Write, cit. nota n. 7, p. 107.

${ }^{33}$ Sobre la estructura Afirmación-Razones-Evidencia-Reconocimiento/Respuesta-Principio, de los argumentos de investigación, ver en general Booth; Colomb; Williams, The Craft, cit. nota n. 3, pp. 120-170 \& 206-207.

${ }^{34}$ Ver, en general, Dunleavy, Authoring a PhD., cit. nota n. 8, pp. 63-74.

${ }^{35}$ Ver, e.g., Germano, From Dissertation, cit. nota n. 8, p. 34.
} 
contraargumento requiere como mínimo dos posiciones, y como máximo tres. ${ }^{36}$ Y utilizar esta estructura en una tesis doctoral, o en un libro, puede resultar extremadamente repetitivo.

Finalmente, está el modelo mixto, que combina las alternativas anteriores. Hay cuatro posibilidades: analítico más descriptivo; analítico más argumentativo; argumentativo más descriptivo, y argumentativo más analítico. El modelo mixto será de utilidad cuando no sea posible o conveniente elegir exclusivamente uno de los otros. En cada una de estas posibilidades, el primer modelo reúne los capítulos de la tesis en grupos; el segundo modelo, en cambio, explica la secuencia de capítulos en cada grupo. ${ }^{37}$

\section{LA FORMA DE UNA TESIS}

Planear una tesis integrada no es tarea fácil. La distribución de su texto debe ser asequible a sus lectores. La macro-estructura de una tesis doctoral o de un libro -vale decir, la sucesión de sus capítulos- debe ser lógica, bien organizada y cumulativa, a fin de permitir la exposición coherente del argumento central. ${ }^{38}$ El problema aquí radica en que la gran mayoría de los investigadores sin experiencia son autores seriales. Van escribiendo capítulo a capítulo en el orden del índice tentativo, empezando -por ejemplo- el tercer capítulo sólo una vez que el segundo está bien definido. Este camino tiene sus peligros. Puede ocurrir que el novel investigador vaya registrando los resultados de su trabajo en el orden en que éstos se presentan en la práctica. Pero en ninguna investigación hay una progresión constante, por lo que el simple registro de su avance producirá una estructura incoherente, que terminará por afectar la línea central de argumentación de la tesis.

Una duda recurrente de los estudiantes de doctorado es la extensión de la tesis. En muchas universidades extranjeras, se establece un límite máximo que asciende hoy a las 100.000 palabras, lo que se traduce en alrededor de 300 páginas. ${ }^{39}$ En la tradición europea mediterránea y latinoamericana, por desgracia, abundan las tesis doctorales interminables -por eso Hernán Corral habla de 400 a 500 páginas, i.e. de 120.000 a 150.000 palabras, cuando se refiere a la extensión

\footnotetext{
${ }^{36}$ Si se sigue el trinomio hegeliano de tesis-antítesis-síntesis.

${ }^{37}$ El modelo descriptivo no se presta para agrupar capítulos. Por lo general, se le utiliza solo. DunLEAvY, Authoring a PhD., cit. nota n. 8, p. 73.

${ }^{38}$ Ver, en general, Dunleavy, Authoring a PhD., cit. nota n. 8, pp. 43-62. Ver, también, RugG; Petre, The Unwritten Rules, cit. nota n. 8, p. 120.

${ }^{39}$ Ver Rugg; Petre, The Unwritten Rules, cit. nota n. 8, 101. Tienen un límite similar, e.g., los programas de doctorado en derecho de las siguientes universidades: Melbourne y Sydney, en Australia; McGill y Toronto, en Canadá; Ámsterdam y Leiden, en los Países Bajos, y Cambridge y Edimburgo, en el Reino Unido.
} 
de éstas. ${ }^{40}$ Como nos recuerda William Germano, antiguo editor de Columbia University Press y de Routledge, un texto de esta magnitud será muy difícil de publicar en el mundo académico de habla inglesa. ${ }^{41}$ Conviene tener un límite determinado como parámetro desde el comienzo de la redacción de la tesis, puesto que es muy fácil escribir en exceso en una investigación. Siempre será más factible rellenar que cortar un texto. Dunleavy recomienda, en la medida de lo posible, escribir un texto que tenga una extensión de aproximadamente tres cuartos del límite máximo exigido. ${ }^{42}$ Esto significa que en una tesis de 100.000 palabras, no debiera pasarse de las 80.000 . Un texto de esta extensión resulta más manejable no sólo para el autor, sino también para el lector. Por lo mismo, será más atractivo a las editoriales que se interesen, eventualmente, por el resultado de la investigación. ${ }^{43}$

Lo normal es que no haya límites mínimos para una tesis doctoral. Informalmente, no obstante, sí los hay. La diferencia que existe en las universidades británicas, entre el MPhil (Magister Philosophae) y el PhD (Doctor Philosophae), sirve para ilustrar lo anterior. Ambos grados académicos son de investigación. Sin embargo, el límite máximo en la tesis de MPhil es, por lo general, de 60.000 palabras, y la exigencia de hacer una contribución original se aplica en forma menos estricta que en el PhD. Una tesis doctoral que se acerque demasiado en su extensión a una tesis de magíster de investigación, corre el riesgo de ser considerada como insuficiente para la obtención del grado de doctor. Esto llevaría a que en la defensa de la tesis se confiera al candidato el grado de MPhil en vez del grado de $\mathrm{PhD} .{ }^{44}$ Como dice, Dunleavy, una tesis doctoral debe verse y sentirse como tal. ${ }^{45}$

Una vez que se tiene la extensión estimada del texto principal, se debe dividir éste en partes. Un principio básico a este respecto, es que la división de la tesis en capítulos debe ser lo más homogénea posible. ${ }^{46}$ La regularidad en el largo de los capítulos permite satisfacer las expectativas del lector, quien sabrá con anticipación la extensión de cada uno de ellos. Adicionalmente, una división en capítulos regulares se ve mejor organizada y da la impresión de

${ }^{40}$ Corral, Cómo hacer, cit. nota n. 2, pp. $99 \& 132$.

${ }^{41}$ Germano, From Dissertation, cit. nota n. 8, pp. 33, $60 \& 97$.

Cf. Cuerda, Cum laude, cit. nota n. 2, pp. 119-20.

${ }^{42}$ Dunleavy, Authoring a PhD., cit. nota n. 8, pp. 45-6.

${ }^{43}$ En el Reino Unido, e.g., la extensión promedio de un libro académico es de 70.000 palabras. DuNLEAVY, Authoring a PhD., cit. nota n. 8, p. 46.

${ }^{44}$ Ver, e.g., RugG; Petre, The Unwritten Rules, cit. nota n. 8, p. 165.

${ }^{45}$ Dunleavy, Authoring a PhD., cit. nota n. 8, p. 47.

${ }^{46}$ Corral, Cómo hacer, cit. nota n. 2, p. 99.

Cf. Cuerda, Cum laude, cit. nota n. 2, p.71. 
que el autor maneja bien su tema. ${ }^{47}$ Para determinar el número de divisiones necesarias en la tesis, se debe tener presente que un capítulo ha de tener una extensión máxima, en la práctica, de 10.000 palabras ${ }^{48}$ Los capítulos que se extienden mucho más allá de este límite son difíciles de organizar internamente, haciendo menos efectiva la argumentación. Por lo demás, los capítulos extensos son más difíciles de transformar en artículos publicables en revistas académicas, los cuales debieran tener -en principio- entre 6.000 a 10.000 palabras. Pero no sólo al determinar la extensión máxima de los capítulos se debe tener en cuenta las expectativas del lector. También se deberán considerar al establecer su límite mínimo. Un capítulo de menos de 6.000 palabras se verá insustancial, y lo confundirá. No está de más decir que estos límites son indicativos. O sea, van a variar caso a caso. Sin embargo, no conviene ir demasiado más allá de los números señalados. No es aconsejable pasar de las 120.000 palabras en una tesis ni bajar de las 80.000 palabras. Tampoco sería aconsejable pasar de las 12.000 palabras en los capítulos ni bajar de las 8.000 palabras. Por lo tanto, si se tiene un capítulo de 18.000 palabras será mejor dividirlo en dos capítulos de 9.000 palabras. ${ }^{49}$

\section{Capítulos y artículos}

Un texto de alrededor de 80.000 palabras, dividido cada 10.000 palabras, da como resultado ocho capítulos. Éste es un buen número, puesto que permite no perder de vista el argumento central de la tesis doctoral o del libro. Con once o doce capítulos, dicho argumento no sólo se diluye para el autor, sino también para el lector. Algunos investigadores sentirán que la regla de los ocho capítulos los restringe mucho y optarán por dividir el texto en dos partes. ${ }^{50}$ Si esto se hace únicamente para intentar manejar una inflación de capítulos, tal solución debe ser desechada de plano. Tiene que haber una razón de fondo que explique la conveniencia de la división de la tesis en dos partes. Tal división debe hacerse únicamente cuando contribuya a darle continuidad a los capítulos. ${ }^{51}$ Éstos se agruparán en: material introductorio, núcleo de la tesis y material de cierre. El núcleo está constituido por todos aquellos capítulos que tienen un alto valor agregado. Son los capítulos que contribuyen a la originalidad del trabajo. Ob-

\footnotetext{
${ }^{47}$ Por el contrario, una tesis cuya estructura es descuidada puede llevar a que el lector pierda confianza en su autor y sospeche que no sólo hay problemas de forma en la tesis, sino que también de fondo.

Ver Murray, How to Write, cit. nota n. 7, p. 63.

${ }^{48}$ Dunleavy, Authoring a PhD., cit. nota n. 8, p. 47.

${ }^{49}$ Dunleavy, Authoring a PhD., cit. nota n. 8, p. 48.

${ }^{50}$ Ver, e.g., Corral, Cómo hacer, cit. nota n. 2, p. 99.

${ }^{51}$ Ver Dunleavy, Authoring a PhD., cit. nota n. 8, pp. 48-49.
} 
viamente, no todos los capítulos pueden pertenecer al núcleo. También habrá una cantidad irreducible de texto que quedará fuera de éste, constituido por: el material introductorio, que prepara la presentación del núcleo, haciéndolo accesible y entendible al lector, y el material de cierre, que integra y reformula lo señalado en el núcleo, ubicándolo dentro del contexto más amplio del área respectiva..$^{52}$ La pregunta de investigación ha de incluirse en la introducción de la tesis. ${ }^{53}$ La afirmación, con la cual comienza el argumento central que da respuesta a la pregunta principal, debiera también incluirse en el capítulo introductorio -hacia al final de éste. Dicha afirmación tendrá que repetirse al comienzo de la conclusión. ${ }^{54}$ Los últimos capítulos que se escribirán en una tesis doctoral o libro serán la introducción y la conclusión.

Respecto a la secuencia de los capítulos, se pueden distinguir dos tipos de modelos. En el primero, el estudiante registra el resultado de su investigación en la forma en que se fue produciendo a lo largo de ésta. ${ }^{55} \mathrm{El}$ índice de la tesis normalmente tendrá dos, tres o incluso cuatro capítulos de análisis de literatura crítica, y sólo cuatro a dos capítulos de trabajo sustantivo, seguidos por una muy breve conclusión final. Este modelo presenta múltiples dificultades. Si la tesis está destinada a hacer una contribución original y ésta se ubica en el núcleo de la tesis, dejar la mitad o menos de la mitad a dicho núcleo no puede ser una buena idea. Tener que leer 100, 150 e incluso 200 páginas sin valor agregado va a colmar la paciencia de cualquier lector, incluyendo a los examinadores de la tesis respectiva, quienes pueden terminar cuestionando su originalidad. ${ }^{56}$ Por desgracia, este modelo se presenta comúnmente en los estudios doctorales. Una de las razones que explican esta circunstancia radica en que les permite a los supervisores o profesores guía de la investigación ir leyendo un tema que no manejan bien junto al doctorando, durante las distintas etapas de redacción de la tesis. Dicho de otra manera: permite al profesor aprender al mismo tiempo que el estudiante. En el segundo modelo, el material introductorio se reduce a dos capítulos. ${ }^{57}$ Se incluirá en éstos todo lo que el lector necesite saber para

\footnotetext{
${ }^{52}$ Ver Dunleavy, Authoring a PhD., cit. nota n. 8, pp. 49-52. Ver, también, Germano, From Dissertation, cit. nota n. 8, pp. 34-5 y 73-5; Воотн; CоLомв; Willams, The Craft, cit. nota n. 3, pp. 232-48, y RugG; Petre, The Unwritten Rules, cit. nota n. 8, pp. 102-103.

${ }^{53}$ Rugg; Petre, The Unwritten Rules, cit. nota n. 8, p. 102.

${ }^{54}$ Ver Bоотн; Cоlomb; Wiluams, The Craft, cit. nota n. 3, pp. 174, 180-181 y 204-205. Ver, también, CuerDA, Cum laude, cit. nota n. 2, pp. 122-123.

${ }^{55}$ Ver Dunleavy, Authoring a PhD., cit. nota n. 8, pp. 53-59. Ver, también, Воотн; Colomb; Williams, The Craft, cit. nota n. 3, pp. 177-178.

${ }_{56}$ Ver, e.g., Germano, From Dissertation, cit. nota n. 8, pp. 62-63, y Cuerda, Cum laude, cit. nota n. 2, p. 61.

${ }^{57}$ Ver Dunleavy, Authoring a PhD., cit. nota n. 8, pp. 51-2 y 60-62.
} 
entender y apreciar el núcleo de la tesis, pero nada más que eso. El autor podrá así desarrollar la pregunta principal en cinco o seis capítulos, quedándole espacio suficiente para presentar el material de cierre en uno o dos capítulos finales.

Los capítulos también deben estar organizados internamente en forma efectiva: esta es la micro-estructura de la tesis. ${ }^{58}$ Dividir un capítulo en secciones es más fácil que dividir la tesis en capítulos, debido a que el primero es más corto y manejable que la segunda. Sin embargo, el esquema elegido para el primer capítulo tiene que servir para todo el resto: la organización interna de cada capítulo debe ser reconocible para el lector, si se quiere satisfacer sus expectativas. Los capítulos están separados por títulos. Cuántas secciones se necesitan en cada capítulo se verá caso a caso. Dunleavy recomienda poner un subtítulo cada 2.000 a 2.500 palabras: divisiones de menos de 2.000 palabras se verán insustanciales. ${ }^{59}$ Además de estas secciones de primer orden, están las secciones de segundo orden dentro de cada subtítulo del capítulo, separadas a su vez por otros subtítulos. Los títulos tienen que ir en forma más destacada que los subtítulos de primer orden. La misma relación se requiere respecto de estos últimos y los subtítulos de segundo orden. Conviene evitar usar un tercer orden de subtítulos, los cuales serán simples agrupaciones de párrafos. Estas secciones, dentro de otras secciones, dentro de otras secciones, dificultarán el control o manejo del argumento central por el autor y no ayudarán en nada al lector. ${ }^{60}$ Junto a la parte constitutiva del núcleo del capítulo, se deben incluir dos secciones breves: una con material introductorio, sin subtítulo, y otra con material de cierre, subtitulada "conclusión". Cada una de estas partes más pequeñas ha de tener una extensión de 200 a 1.000 palabras. ${ }^{61}$ Lo señalado anteriormente para la micro-estructura de una tesis doctoral o de un libro, será aplicable a la estructura de un artículo académico. ${ }^{62}$

Dos errores son comunes al momento de organizar la estructura interna de un capítulo o artículo. ${ }^{63}$ El primero es que falte organización. Ocurre cuando se crean secciones que son notoriamente más largas que otras, aun cuando se siga un orden homogéneo de títulos y subtítulos en todos los capítulos. Por ejemplo, que la sección correspondiente al primer subtítulo principal tenga 1.000 palabras, mientras la sección correspondiente al segundo subtítulo principal tenga 9.500 palabras. También se presenta este error, en una tesis

\footnotetext{
${ }^{58}$ Ver, en general, Dunleavy, Authoring a PhD., cit. nota n. 8, pp. 76-79.

${ }^{59}$ Dunleavy, Authoring a PhD., cit. nota n. 8, p. 77.

${ }^{60}$ Cf. Corral, Cómo hacer, cit. nota n. 2, p. 99.

${ }^{61}$ Dunleavy, Authoring a PhD., cit. nota n. 8, p. 79.

${ }^{62} \mathrm{O}$ de una tesis de magíster.

${ }^{63}$ Ver Dunleavy, Authoring a PhD., cit. nota n. 8, pp. 79-84.
} 
doctoral o libro, cuando se organizan distintos capítulos de manera diferente. Esto sucede cuando no se utiliza el mismo sistema de títulos y subtítulos a lo largo del texto. La mayoría de quienes empiezan a investigar -particularmente, los doctorandos- escriben un capítulo a la vez, y con el transcurso del tiempo pueden cambiar sus ideas respecto a títulos y subtítulos. Hay que ser consistente en el uso de títulos y subtítulos, sin caer en un esquema inflexible. La estructura elegida tiene que responder a la naturaleza de cada capítulo, y esto no se consigue aplicando mecánicamente una organización disociada del contenido respectivo. El segundo error es sobre organización, lo que se produce cuando se tienen demasiados niveles de subtítulos. ¿Ejemplo? Que se divida un capítulo en once subtítulos de primer orden, los cuales se dividan, asimismo, en tres o cuatro subtítulos de segundo orden, y éstos se dividan, a su vez, en subtítulos de tercer orden. Un texto así de fragmentado, sólo conseguirá confundir al lector. La conclusión que éste sacará es que al autor no le interesa que su texto sea asequible. El argumento central de la tesis se verá afectado, puesto que dicho esquema terminará por privarlo de cohesión. Será mejor, por tanto, no pasar de los subtítulos de primer y segundo orden, evitando agregar subdivisiones de tercer orden.

\section{CONCLUSIÓN}

Escribir una tesis es responder a una pregunta: pero no de cualquier manera. Un investigador debe pensar como un escritor, y éste quiere ser leído. De ahí que la consideración hacia su eventual lector ocupe un lugar central en todo el proceso de investigación. Se comenzará por elegir un tema y formular una pregunta, destinada a resolver un problema relacionado con aquél. Después vendrá la elaboración de la bibliografía y del índice tentativos. Con este plan de trabajo, el autor de una tesis doctoral o de un libro tendrá que elegir una macroestructura que le permita dar una respuesta coherente a la pregunta principal de su investigación. Tendrá, asimismo, que optar por una micro-estructura que le dé consistencia y homogeneidad a los capítulos en que ha dividido su trabajo. El autor de un artículo académico podrá prescindir de la macro-estructura, pero no de la micro-estructura. En uno u otro caso, la pregunta deberá estar bien planteada y la respuesta bien argumentada. De lo contrario, el autor fracasará en su intento por comunicar sus hallazgos.

Articular una respuesta efectiva a la pregunta principal de una investigación, que constituya una contribución original, requiere cierto oficio. Un aspecto fundamental en este proceso es el manejo de las expectativas del lector. Éstas se crean, para bien o para mal, en el material introductorio de la tesis y deberán necesariamente satisfacerse en su núcleo y, particularmente, en su material de cierre. Ayuda a adquirir este oficio el ejercicio del mismo: para investigar, hay que empezar a hacerlo. Y seguir haciéndolo. 


\section{BiBLIOGRAFÍA}

Álvarez Undurraga, Gabriel, Curso de investigación jurídica, Editorial LexisNexis, Santiago de Chile, 2005.

Arellano García, Carlos, Métodos y técnicas de la investigación jurídica, Editorial Porrúa, México D.F., 1999.

BaIN, Ken, What the Best College Teachers Do, Harvard University Press, Cambridge-Massachusetts, 2004.

Воотн, Wayne; Colomb, Gregory; Williams, Joseph, The Craft of Research, University of Chicago Press, Chicago, 2008.

Corral Talcianı, Hernán, Cómo hacer una tesis en derecho, Editorial Jurídica de Chile, Santiago, 2008.

Cuerda Riezu, Antonio, Cum laude. Guía para realizar una tesis doctoral en derecho, Editorial Tecnos, Madrid, 2008.

Dunleavy, Patrick, Authoring a PhD. How to Plan, Draft, Write and Finish a Doctoral Thesis or Dissertation, Palgrave Macmillan, Nueva York, 2003.

Eco, Umberto, Cómo se hace una tesis. Técnicas y procedimientos de investigación, estudio y escritura, Editorial Gedisa, Barcelona, 1993.

Elgueta, María Francisca; Palma, Eric, La investigación en ciencias sociales y jurídicas, Universidad de Chile, Santiago, 2008.

Germano, William, Getting it Published, University of Chicago Press, Chicago, 2008 cago, 2005.

Hernández Estévez, Sandra; López Durán, Rosalío, Técnicas de investigación jurídica, Oxford University Press, México D.F., 2002.

Herrera, Enrique, Práctica metodológica de la investigación jurídica, Editorial Astrea, Buenos Aires, 2002.

Lara Sáenz, Leoncio, Procesos de investigación jurídica, UNAM, México D.F., 1991.

Martínez Pichardo, José, Lineamientos para la investigación jurídica, Editorial Porrúa, México D.F., 1998.

Murray, Rowena, How to Write a Thesis, Open University Press, Maidenhead, 2009.

Ramos Núñez, Carlos, Cómo hacer una tesis en derecho y no envejecer en el intento, Gaceta Jurídica, Lima, 2007.

RugG, Gordon; Petre, Marian, The Unwritten Rules of PhD Research, Open University Press, Maidenhead, 2009.

SAVATER, Fernando, Instrucciones para olvidar el Quijote, Editorial Santillana, Madrid, 1995. 
Scavone, Graciela, Cómo se escribe una tesis, Editorial La Ley, Buenos Aires, 2002.

WiTKER, Jorge, Cómo elaborar una tesis en derecho, Editorial Civitas, Madrid, 1991. 